\title{
Simulation in plastic surgery: Features and uses that lead to effective learning
}

\author{
C P G Nel, ${ }^{1}$ MB ChB, MHPE, FC Plast Surg (SA), MMed (Plastic Surgery), PhD; G J van Zyl, ${ }^{2}$ MB ChB, MFamMed, PhD; \\ M J Labuschagne, ${ }^{3} \mathrm{MB} \mathrm{ChB}, \mathrm{MMed}($ Ophthalmology), $\mathrm{PhD}$ \\ ${ }^{1}$ Department of Plastic Surgery, Faculty of Health Sciences, University of the Free State, Bloemfontein, South Africa
${ }^{2}$ Faculty of Health Sciences, University of the Free State, Bloemfontein, South Africa
${ }^{3}$ School of Biomedical Sciences, Faculty of Health Sciences, University of the Free State, Bloemfontein, South Africa
}

Corresponding author: C P G Nel (drcnel@gmail.com)

\begin{abstract}
Background. Increased competition for surgical exposure and practice, smaller teaching platforms and shorter training times have an impact on the quality of training and competence of plastic surgery registrars. Demands for accountability and minimising patient risks are the driving forces for incorporating simulation in healthcare education. We addressed the problem of whether the features and uses of simulation would enhance postgraduate plastic surgery education and training and ensure more effective learning.

Objective. To identify and describe: (i) how simulation impacts on student learning; therefore, how the effectiveness of learning may be enhanced in postgraduate and/or plastic surgery education and training; and (ii) which features and uses of simulation have the potential to enhance learning in plastic surgery.

Methods. A descriptive design was used for the study. Data were collected by means of semi-structured interviews with 8 national and international role players in simulation.

Results. The results indicated a positive outcome of simulation, as it provides, e.g. a non-threatening environment for learning and improves clinical competency, ensuring an increase in patient safety. The features and uses of simulation render it an excellent method to enhance learning effectiveness at different cognitive levels and to fulfil a specific role in integrated and holistic training, while providing opportunities to practise specific skills. The lack of clinical opportunities can be addressed, and more clinical exposure and practice will result in fewer medical errors.

Conclusion. Simulation-based education in postgraduate plastic surgery education and training proved to be an effective teaching-learning method, which provides solutions to current deficiencies, hindrances and gaps in health professions education. The research question was answered and the use of simulation is recommended to enhance plastic surgery education and training and promote safe patient care.
\end{abstract}

Afr J Health Professions Educ 2021;13(1):29-35. https://doi.org/10.7196/AJHPE.2021.v13i1.1181

Evidence of the role of simulation in medical education has emphasised the use of simulation technology over the past number of decades in an effort to increase learner knowledge, to provide students with controlled and safe practice opportunities, and to shape the acquisition of doctors' clinical skills. ${ }^{[1-3]}$ Simulation is becoming an integral part of medical education at all levels, ${ }^{[1-3]}$ as medical education, for various reasons, has fast become subject to radical and innovative changes.

Many major shifts in medical education methods are due to changes in the delivery of healthcare. According to Issenberg et al. ${ }^{[3]}$ in the USA, for example, the pressures of managed care shape the form and frequency of hospitalisation, 'resulting in higher percentages of acutely ill patients and shorter in-patient stays'. Medical students, therefore, have fewer opportunities to assess patients with a wide variety of diseases and physical findings, while reductions in physician remuneration due to shrinking financial resources constrain the educational time that doctors in training receive. ${ }^{[4]}$ Consequently, at all educational levels, doctors find it increasingly difficult to keep abreast of skills and topics they need to practise successfully. ${ }^{[4]}$

Issenberg et al. ${ }^{[3]}$ identify 5 factors that contribute to the increased use of simulations in medical education, i.e. lack of clinical teaching opportunities and therefore less patient material due to changes in healthcare delivery; new technologies for diagnosis and management; assessing professional competence; medical errors, patient safety and team training; and the role of deliberate practice. ${ }^{[4]}$

Surgical training in the 21 st century is characterised by an increasingly objective, standardised approach using equipment, such as simulators, to optimise patient safety, surgical care and hospital resources, and minimise errors. ${ }^{[5]}$ The driving forces behind these factors are developments in medical error statistics, evidence-based medicine and fewer attending hours. Through increased accuracy, simulation can improve results and lower risk and procedure costs because of fewer procedures and less operating room time.$^{[5]}$ Simulation during training allows students ample opportunity to hone their skills and competencies in safe, no-risk circumstances. Insufficient and inefficient clinical teaching has stressed the need for strategies to improve clinical education, including the use of simulation ${ }^{[5]}$ Simulation-based medical education is an educational method that makes use of simulation to bridge the gap between theory and practice. ${ }^{[6]}$ Regarding medical simulation, the word simulation means 'imitation of the operation of a real-world process or system over time. ${ }^{\text {?] }}$

Over the past 30 years, new technologies in medicine have revolutionised patient diagnosis and care. Examples are the development of flexible sigmoidoscopy and bronchoscopy, and minimally invasive surgery, including laparoscopy, and robotics for orthopaedics, urology 
and cardiology. The benefits of these innovations include reduced postoperative pain and suffering, shorter hospitalisation and earlier resumption of normal activities, as well as significant cost savings. ${ }^{[8]}$

These newer techniques, however, demand psychomotor and perceptual skills that differ from traditional approaches, and these innovative methods may be associated with a higher complication rate than traditional practices. ${ }^{[9]}$ Haluck et al. ${ }^{[10]}$ maintain that these 'newer technologies have created an obstacle to traditional teaching that included hands-on experience. For example, endoscopy requires guiding one's manoeuvres in a three-dimensional environment by watching a two-dimensional screen, requiring the operator to compensate for the loss of binocular depth cue with other depth cues.' One of the corollaries to these new techniques was the introduction of simulation technology in the training and assessment of students. Research indicates that training programme directors emphasised that virtual reality and computer-based simulations have become indispensable technological tools in clinical education. ${ }^{[10]}$

The Accreditation Council for Graduate Medical Education (ACGME) in the USA, in an endeavour to ensure and improve the quality of graduate clinical medical education and to attain a higher level of effectiveness, listed 6 domains of clinical medical competence. ${ }^{[8]}$ Postgraduate programmes should provide educational experiences, which ensure that graduates demonstrate competence in ACGME project outcomes, i.e. patient care; medical knowledge; practice-based learning and improvement; interpersonal and communication skills; professionalism; and system-based practice. ${ }^{[1]}$ These are the educational experiences that benefit most from simulation in the light of a lack of patients and clinical exposure.

Miller ${ }^{[12]}$ proposed a framework (Miller's pyramid), which argues that a medical learner's clinical skills should be assessed at four levels: (i) knows (knowledge) - recall of facts, principles and theories; (ii) knows how (competence) - ability to solve problems and describe procedures; (iii) shows how (performance) - demonstration of skills in a controlled setting; and (iv) does (action) - behaviour in real practice. ${ }^{[4]}$ Simulation technology is increasingly being used in each domain of competence to assess the first three of Miller's levels of learning because of its ability to programme and select learning-specific findings, conditions and scenarios to provide standardised experiences to all examinees and to include outcome measures that yield reliable data. ${ }^{[13]}$ The research question was whether the features and uses of simulation would enhance plastic surgery education and training and ensure more effective learning.

\section{Methods}

The research included data collected by means of semi-structured interviews and a Delphi process. This article focuses on outcomes of the interviews. An article dealing with the Delphi part of the study has been published in AJHPE. ${ }^{[14]}$

Elements of grounded theory came into play to describe features and uses of simulation, and to relate why simulation lends itself perfectly to be included in educational programmes. Grounded theory (inductive approach) was used to develop recommendations to promote learning in postgraduate plastic surgery education and training, as a grounded theory deals with discovering, developing and verifying by means of systematic data collection and data analysis pertaining to a phenomenon. ${ }^{[15]}$

The study focused on the opinions and perspectives of medical and healthcare professionals regarding the features and uses of simulation, and whether and how simulation as an education and training method might influence student learning. The study was aimed at developing recommendations to enhance the effectiveness of learning in postgraduate plastic surgery education and training by employing simulation as one of the methods used.

\section{Semi-structured interviews}

Semi-structured individual interviews were used to explore key national and international role players' opinions and perceptions on simulationbased medical education, aimed at investigating and establishing clarification on simulation in postgraduate plastic surgery education and training. The features and uses of simulation that might prove effective in postgraduate plastic surgery were thus determined. The researcher conducted the interviews because he is a skilled interviewer and was familiar with the topic; he knew how to phrase the questions, as he was aware of the frame of reference of the interviewees and the goals of the study. It was also important to concentrate on the responses and monitor the coverage of the topic - which would have been impossible for another interviewer. ${ }^{[16]}$ An interview guide (Table 1), developed by the author on the basis of a literature review, was used. Occasionally,

Table 1. Guide for semi-structured interviews with national and international role players

\section{Question}

1. What experience with/exposure to simulation in the field of health education have you had in general? Briefly describe your experience with/exposure to simulation in terms of the type of simulation, your role, duration of involvement.

2. Are you currently involved in simulation and/or postgraduate education and training? In what context are you involved?

3. Does/can simulation influence student learning in postgraduate education and training? In what regard?

4. How can effectiveness of learning be enhanced in postgraduate and/or plastic surgery education and training (in the areas of knowledge, skills, clinical competence, professional conduct)?

5. Can simulation be used to enhance student learning at different cognitive levels? (Will the student only use simulation to remember knowledge/or understand/or apply/or analyse/or evaluate/or create new concepts and ideas?)

6. Which types of simulation or simulation modalities might lead to effective learning?

7. Which features and applications of simulation in postgraduate or plastic surgery education and training will lead to more effective learning?

8. Does simulation have: (i) a contribution to make to postgraduate education; (ii) a role to play in postgraduate education; or (iii) a specific value to add to postgraduate education?

9. What would your main consideration be if you decided to include simulation in your teaching and training programme?

10. If you have to guide a team of experts tasked to develop a curriculum with simulation as one of the training/learning methods, which important guidelines would you put forward?

11. Do you wish to make any recommendations that may be used in compiling guidelines on simulation for postgraduate plastic surgery?

12. Are there any recommendation(s) you would like to make when considering including simulation in specialist training?

13. Will you please share: (i) some of the lessons learnt regarding the implementation of simulation in a curriculum; and (ii) the biggest challenge in implementing simulation in training? 
additional questions arose during the semi-structured interviews; the data thus collected were included in the research. Data on questions 3, 4 and 7 of the interview guide are reported in this article.

\section{Unit of analysis}

National and international role players in simulation and postgraduate education were requested to participate in the semi-structured interviews. Eight participants were invited to participate, all of whom accepted the invitation. Four interviewees were from the USA, Canada and the UK and 4 from South Africa (SA). They were directors of simulation units, clinical heads of clinical medical departments, programme directors of medical and nursing programmes, and education management specialists, researchers and representatives from the simulation industry. Written consent was obtained from all participants.

\section{Data collection and analysis}

The author conducted individual interviews with 8 participants, based on an interview guide. The interviews were audio-recorded, transcribed and checked by an independent person who was not part of the study. Field notes taken during the interviews contributed to the data. The data were analysed using the grounded theory approach, which requires continuous comparison of data, following the data analysis steps of coding, categorisation and theory generation. ${ }^{[17]}$ Theory building occurred by finding patterns in the data, which continued until saturation of data was reached. ${ }^{[18]}$ As more data were collected and re-reviewed, codes were grouped into concepts, and then into categories. These categories formed the basis for new theory, and were compared with data collected during the literature study. Data saturation was reached when no more ideas came to the fore.

\section{Reliability and trustworthiness}

Reliability was ensured by making use of an explorative study (with 2 individuals who were involved, had a sound knowledge of simulation and complied with the selection criteria), determining strict criteria for sampling, using the carefully constructed interview guide, as well as an interview process that was audio-taped and carefully described. ${ }^{[19]}$ Trustworthiness of the interviewing process was ensured by involving voluntary interviewees with a clear understanding of what the interviewer expected from them, and using open-ended questions, as well as the transcription and verification of data. Scientific record keeping ensured dependability ${ }^{[19]}$

\section{Ethical approval}

Ethical approval to conduct the research was obtained from the Ethics Committee of the Faculty of Health Sciences, University of the Free State, Bloemfontein, SA (ref. no. ECUFS 122/2015).

\section{Results}

Data collected by means of questions 3, 4 and 7 of the semi-structured interviews are reported in this article. Data were analysed, findings were summarised and qualitative perspectives are shared on the influence of simulation on student learning (Table 2) and how the effectiveness of learning can be enhanced (Table 3). The features and uses of simulation that may enhance learning in postgraduate education and training served as basis for a number of recommendations to enhance the effectiveness of learning in postgraduate plastic surgery education and training (Table 4). Quotes from
Table 2. Influence of simulation on student learning in postgraduate education and training

1. Simulation as a learning strategy

Simulation influences student learning in different ways:

- Simulation is a very specific learning and education strategy that can be described as a holistic process that ensures meaningful learning

- 'To be effective it needs to be an important component in the curriculum.' (S1)

- 'Effectiveness can only be enhanced if there is proper integration.' (S1)

- Simulation is based on an adult learning strategy, as it is built on adult learning principles that can be combined in different ways

- 'Different people learn in different ways.' (C3)

- Simulation can replace other educational strategies, e.g. theoretical lectures, by bringing simulated case engagement into the normal learning strategy

- 'Simulation will trigger more excitement.' (D1)

- 'Students will be more focused and remember more'. (D1)

- 'It gives students the opportunity to learn hands-on.' (C1)

2. Motivation to make a difference

Simulation of rare clinical cases or life-threatening, important scenarios is motivational:

- '... students can be motivated to make a difference in a patient's life.' (C1)

3. Importance of preparation

Theoretical grounding and preparation will influence student learning:

- 'Students may attend a lecture, e-learning programme, read articles, do a self-test before visiting the simulation lab, which ensures that learning is more meaningful, and promotes deep learning and commitment.' (D1)

Identify own shortcomings, evaluate own level of competence against own tempo:

- 'Students must be prepared when they come to the simulation lab.' (D2)

4. Learn by repetition

Practising non-technical and technical skills will influence student learning:

- 'Giving students the opportunity to practise in the simulation lab so that they are more competent when performing certain procedures on a patient.' (C1, D1)

\section{Group simulations}

Simulation scenarios that include different health professionals will foster and enhance learning:

- 'Debriefing provides students the opportunity not only to identify their own shortcomings, but also those of the individual group members and of the group as a whole.' (C1)

Learn to work in groups and that communication among them is of great importance:

- 'You have to have small-group engagement.' (S1)

6. Level of competence

The aim of simulation is to attain and sustain a certain level of competence or expertise:

- Ensure that the health professional/student maintains the same level of competence after the initial mastering of the procedure

- 'Attaining some sort of competence level quicker before going to the patient.' (C2)

- 'Competent = bare minimum (knows/knows how).' (C2)

- 'Proficient $=$ level of skill above minimum (shows/does).' (C2)

- 'As you practise a skill more, you become proficient $=$ on your way to mastering.' (C2) 
Table 3. Enhancing the effectiveness of learning in postgraduate and/or plastic surgery education and training by introducing simulation

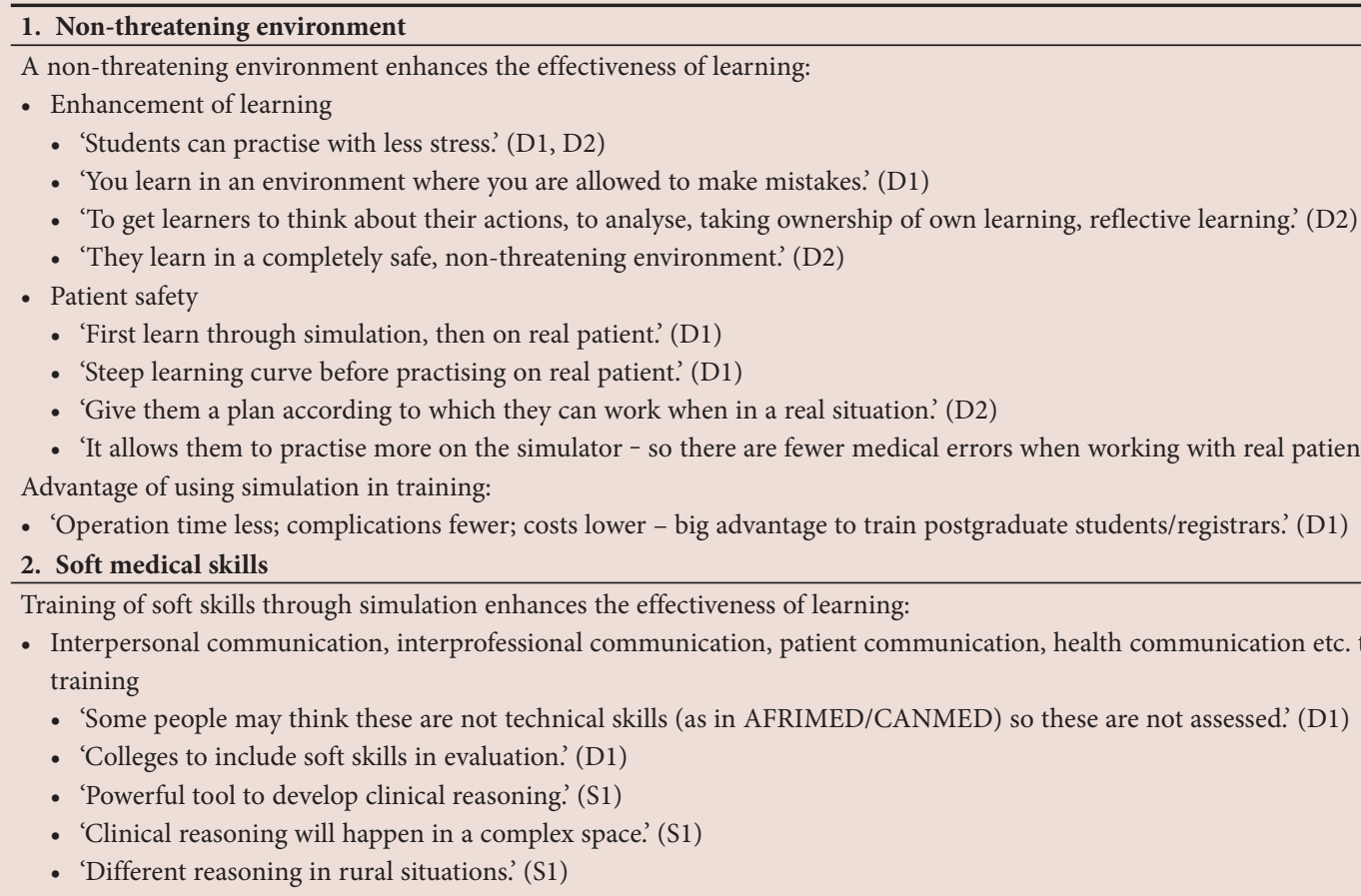

\section{Deliberate practice}

Deliberate practice enhances the effectiveness of learning:

- Bench models may be used to practise psychomotor skills by repetition or deliberate practice of specific procedures until students feel safe

- 'To make deliberate practice more successful, students may use a tick-sheet to test themselves.' (C2)

- 'To make deliberate practice more successful, the student must be accompanied by consultant/educator'. (D1)

- 'Student must know what he/she is doing correctly' (D1)

- 'There must be scheduled times for practice, as well as for feedback.' (D1)

- 'Registrars are adult learners and identify skills that need more practice.' (D1)

\section{Feedback}

Feedback enhances the effectiveness of learning:

- Constructive feedback

- 'Can be according to a debriefing manner: first focus on the positive, then focus on things which he/she should do in another way without negative critique.' (D1)

- 'Give feedback directly after simulation/time slot; in writing or on video.' (D1)

- 'Feedback to be planned, structured according to a template or may be more personal.' (D1)

- 'For non-technical skills use the debriefing method - look at what was good; discuss what can be different.' (D1)

- 'For technical skills - use a tick-sheet; it will allow for more constructive feedback and certain skills can be revisited.' (D1)

- 'Give feedback to students so that they can correct themselves.'

- Feedback and assessment with a view to enhancing the effectiveness of learning

- 'Formative feedback will help students to prepare for assessment and examination; then they will have time to correct their mistakes.' (D1)

- 'Timely feedback before assessment can help the student to lower stress levels.' (D1)

- 'Feedback on quality of operations can offer opinion where the registrar is safe; by using easy scenarios evaluate whether the registrar executes the technique in a good/proper way and/or quick enough - this is a good learning opportunity.' (C1)

- 'Students to be observed on a continuing basis.' (C2)

- 'Student can be observed during a skill performance' (C2)

\section{Debriefing}

Debriefing enhances the effectiveness of learning:

- The process of debriefing gives more insight

- 'Debriefing is another way to learn.' (C1)

- 'It is the debriefing aspect that affects learning.' (D2)

- 'Do not use debriefing as a teaching opportunity, let learners think how they can improve'. (D2)

- 'Observation of skills, then debriefing after that, improves practical skills.' (C2) 
Table 3. (continued) Enhancing the effectiveness of learning in postgraduate and/or plastic surgery education and training by introducing simulation

6. Realism or fidelity

Realism of fidelity enhances the effectiveness of learning:

- The degree of realism of a scenario or patient influences the effectiveness of learning: 'In the beginning, students do not believe in the simulation scenario; later you can observe the "overcoming of disbelief" - the suspension of disbelief - they think it is a real patient: that leads to more effective learning than using a paper case.' (D2)

\section{Repetitive practice}

Repetitive practice enhances the effectiveness of learning:

- For learning to take place, a medical or practical situation is necessary

- 'You have to practise on a continuous basis - do it repeatedly over the time span of a month or year.' (C1, D1)

- 'It must become a natural action - you learn by repeating - repeat soon after the first practice; 1 week; after weeks; months.' (C1)

8. Training platform

The training platform enhances the effectiveness of learning:

- As training platforms may become smaller in certain disciplines, simulation offers opportunities for learning

- 'Platform varies from rural situation to complex.' (S1)

\section{Assessment}

\section{Assessment enhances the effectiveness of learning:}

- Evaluation of clinical skills in summative assessment of registrars plays a role in effective learning

- 'In the field of specialists, assessment should include well-defined objectives and competencies.' (C2)

- 'Assess levels for competence.' (C2)

- 'Assessment to be reliable and statistically sound; use tick-sheet.' (C2)

- 'Use simulated patients for clinical examinations.' (S2)

interviewees' responses are indicated in inverted commas, followed by a code number assigned to each of the participants.

Simulation influences student learning (Table 2), as it substitutes other learning strategies; it supports adult learning principles, as it requires students to prepare, placing a responsibility on them as adult learners; it provides self-confidence and skills, motivating students to confront life-threating situations, making a difference to a patient's life; it provides the opportunity to learn by repetition, to work individually or in groups; it fosters communication; and it ensures that the student attains and sustains a specific level of competency.

Interviewees' opinions on how simulation can enhance the effectiveness of learning are indicated in Table 3 and emphasise the role of simulation as a non-threatening learning method that enhances the effectiveness of learning. Students can practise with less stress in a completely safe environment before working with real patients; this highlights the advantages of training using simulation. Simulation also enhances the effectiveness of learning by fostering interpersonal, interprofessional patient communication, communication regarding health and reasoning skills. Through deliberate, as well as repetitive practice, learning is enhanced (Table 3). The debriefing aspect offers another way of learning and allows students to decide on selfimprovement. Authentic scenarios help students to learn more effectively than when using paper cases. The assessment opportunities of simulation improve student learning.

Recommendations to enhance the effectiveness of learning in postgraduate plastic surgery education and training are offered in Table 4. To apply the unique features and uses of simulation in a correct manner influences the effectiveness of learning in a positive way.

\section{Discussion}

The third and fourth semi-structured interview questions addressed the effect of simulation on student learning and how learning may be enhanced by simulation as a learning method in postgraduate and/or plastic surgery education and training. The opinion was that simulation does enhance the effectiveness of learning as far as the mastery of knowledge, skills, clinical competence and professional conduct is concerned.

The findings of data gathered during the semi-structured interviews were compared with perspectives gained from the literature review. Key outcomes of this research were the identification of the features and uses of simulation, and how simulation might be applied to enhance the effectiveness of learning in plastic surgery. As specific features and uses of simulation influence learning positively, these should be maximised in plastic surgery education. The results of the study provided ample evidence that simulation improves teaching and learning in medical (surgical) postgraduate education, as is evident from the following research.

According to Issenberg et al., ${ }^{[4]}$ 'traditional medical training has focused on individual learning to care for individual patients. Medical education has neglected the importance of teamwork and the need to develop safe systems. The knowledge, skills and attitudes needed for safe practice are not normally acquired, nor are they required, as part of medical education. Simulation is an appropriate method for team training - a prerequisite for interprofessional healthcare required of modern medical education.

Simulation offers the possibility of a cyclic learning dimension structure, i.e. a safe, purposefully planned learning environment, including variations of learning strategies/methods and the opportunity to select material offering different applicable learning opportunities, and ensuring a unique learning experience where the learning can be evaluated by the registrar or feedback/debriefing can be done by a consultant to achieve competence, or to re-plan and/or deliberately practise specific, identified learning units.

Deliberate practice, not just time and experience in clinical settings, is key to the development of medical clinical competence. ${ }^{[4]}$ Deliberate practice involves '(a) repetitive performance of intended cognitive and psychomotor skills in a focused domain, coupled with (b) rigorous skills assessment that provides learners with (c) specific, informative feedback, 
Table 4. Recommendations to enhance the effectiveness of learning in postgraduate plastic surgery education and training by applying the unique features and uses of simulation

\section{Environment}

The provision of a controlled non-threatening environment to registrars will unlock the opportunity to learn more effectively:

- Simulation has the ability to create a safe environment, enabling registrars to detect problems and patient care errors in a non-threatening way of learning, fostering reasoning skills and thought processes; learning takes place where it is acceptable to make mistakes and causes less stress

- The simulation environment is open to accommodate a process that can change from a uni- to a multipurpose process where learning can be based on a single objective or on multiple objectives; learning could span different cognitive levels and include variations from superficial to deep learning

- A simulation scenario may be changed or adapted to a more complex scenario, where learning could take place at a entirely different level of competence and applicability

\section{Curriculum}

The integration of simulation in the curriculum of a postgraduate plastic surgery training programme will offer registrars the opportunity to learn more effectively:

- Simulation should be integrated in the curriculum and training schedule and be directed by guidelines for teaching through simulation; the role of simulation should be clearly stated in curriculum documents and how it will form part of the registrars' performance management

- Teaching and learning strategies should be aligned with educational goals and learning outcomes and should be adaptable to the learning situation

- Simulation offers the opportunity for large-group training by developing scenarios for multiprofessional teams, where individual and group learning can take place with an opportunity for debriefing and constructive feedback; individualised learning according to the registrars' learning needs set to specific standards should be offered

\section{Clinical teaching and learning}

Simulation should offer registrars opportunities for clinical learning to be more effective:

- Registrars' engagement in deliberate practice should take place according to set learning outcomes based on real clinical problems in simulated settings; the realism of clinical problems and the hands-on experience should help them to master clinical outcomes and to transfer knowledge, skills and competencies to real clinical settings

- Learning outcomes will enhance the effectiveness of learning and processes must be in place to ensure they are met

- Smaller learning units can give registrars the opportunity to master learning outcomes at their own pace, ensuring intrinsic motivation and fostering deep learning

- Effective learning is enhanced when registrars practise clinical skills across a wide range of difficulty levels; scenarios set on different levels of difficulty ensure that learning takes place at different cognitive levels

- By offering registrars the opportunity to engage in repetitive practice in a safe environment will give them the challenge to correct and hone their clinical skills and competencies

Feedback

Registrars should approach simulation opportunities in a different way when accompanied by consultants, who give feedback; they will experience scenarios as a direct learning opportunity; problems can be corrected immediately - they should see it as teaching them purposefully and deliberately:

- Feedback should be planned, formally scheduled and be an integral part of the training programme

- Feedback should be built into simulations, or presented at scheduled times, on video or electronic media

- Registrars should use the opportunity of feedback to correct themselves by taking notice of feedback on accuracy and timing

- Constructive feedback should drive decisions as far as preparation for final assessment

Technology

Technology should be seen as offering endless possibilities to enhance learning for registrars:

- Technical skills, non-technical skills and the softer skills can be explored for learning at different levels by adding or changing scenarios

- Sharing facilities between institutions will be beneficial, as registrars can see or use a whole spectrum of simulations; standardisation of outcomes is possible, as well as opportunities for quality learning and learning of new skills

that results in increasingly (d) better skills performance in a controlled setting. ${ }^{[4]}$ Simulation is the ideal way to ensure deliberate practice, regardless of whether patient material is available or not. Research emphasises the importance of repetition for clinical skills acquisition and maintenance, ${ }^{[20]}$ and research evidence clearly shows that high-fidelity medical simulations facilitate learning. ${ }^{[4]}$

Simulation-based education allows students to practise and acquire patient care skills in a controlled and safe learning environment. Feedback to students, the opportunity for deliberate and repetitive practice, multiple learning strategies, individualised learning within a controlled environment, and the opportunity for hands-on experience foster students' self-confidence and play a cardinal role in mastering educational outcomes. ${ }^{[8]}$

To revolutionise medical education, an increased efficiency of education by standardising the curriculum, an individualisation of education and a shift from time-based training to competency-based training are essential. ${ }^{[21]}$ Residents (registrars/specialists in training) may receive little guidance in terms of the knowledge, competencies, skills and attitudes that they are expected to acquire during residency. Surgical training in the 21st century is characterised by an increasingly objective, standardised approach using equipment such as simulators to optimise patient safety, surgical care and hospital resources, and to minimise errors. ${ }^{[5]}$ The driving forces behind this are developments in medical error statistics, evidence-based medicine and fewer attending hours. Through increased accuracy, simulation can improve results and also lower risk and procedure cost because of fewer procedures and less operating room time. ${ }^{[5]}$

Simulation can play an important role in postgraduate education; however, it cannot totally substitute education involving real patients in genuine settings. 


\section{Conclusion}

From the findings of this research, it is clear that simulation can be introduced as a teaching method and a learning opportunity for residents to improve plastic surgery education and training. To ensure success, however, clear recommendations on how simulation can enhance effective learning, and a description of the role and value of simulation based on scientific research, should be available. This requires the development of guidelines for teaching through simulation as part of training programmes for evidence-based plastic surgery education/practice.

Research is required to enhance the role of simulation in plastic surgery training, ${ }^{[22,23]}$ and this study made a contribution in that regard by identifying why and how simulation can improve the effectiveness of postgraduate and/ or plastic surgery teaching and learning. 'Simulation has the potential to play an integral role in developing better and safer health care services for patients worldwide, avoiding risk and providing real-life opportunity for students to hone their skills. ${ }^{[22,23]}$ The features and uses of simulation discussed here will contribute to and lay the foundation for more effective learning in plastic surgery education and training in the future. The novel contribution made by this study entails the compilation of the advantages simulation holds for medical education, with special reference to postgraduate plastic surgery education, and the detail in which this teaching-learning method is expounded.

Declaration. The research for this study was done in partial fulfilment of the requirements for CPGN's PhD degree at the University of the Free State.

Acknowledgements. The authors gratefully acknowledge the assistance received from the participants in the overall $\mathrm{PhD}$ study, who were willing to take part in the pilot testing and quality control of the Delphi questionnaires. We also acknowledge the role players who participated in the explorative study that conducted the interviews, and specifically the interviewees involved in the research reported in this article, for their valuable contributions and their time in participating in individual semi-structured interviews. We thank Prof. G Joubert, Head: Department of Biostatistics, Faculty of Health Sciences, University of the Free State, for her advice during the early stages of the research, and for the protocol development and quality assurance of the study; and Dr M J Bezuidenhout, University of the Free State, for support with regard to the scientific formulation and language editing of the publication, as well as editing of the references.

Author contributions. CPGN designed the study, wrote the protocol, collected data and performed the analysis, interpreted data and wrote the manuscript. GJvZ and MJL were supervisors of the study, reviewed the protocol and manuscript and contributed substantially to the conceptualisation, design, analysis and interpretation of data and scientific content. All authors approved the final version of the manuscript.
Funding. This research was partially funded by a grant from the Health and Welfare Sector Education Training Authority (HWSETA).

Conflicts of interests. None.

1. Fincher RME, Lewis LA. Simulation used to teach clinical skills. In: Norman CPM, van der Vleuten CPM, Newble DJ, eds. International Handbook of Research in Medical Education, Part 1. Dordrecht, The Netherlands: Kluwer Academic Publishers, 2002:91-95.

2. Gaba D. Human work environment and simulators. In: Miller RD, ed. Anaesthesia. 5th ed. Philadelphia: Churchill Livingstone, 2000:236.

3. Issenberg SB, McGraghie WC, Hart IR, et al. Simulation technology for health care professional skills training and assessment. JAMA1999;282(9):861-866. https://doi.org/10.1001/jama.282.9.861

4. Issenberg SB, McGaghie WC, Petrusa ER, et al. Features and uses of high-fidelity medical simulations that lead to effective learning. Med Teach 2005;27(1):10-28. https://doi.org/10.1080/01421590500046924

5. Rosen JM, Long SA, McGrath DM, Greer SE. Simulation in plastic surgery training and education: The path forward. Plast Reconstruct Surg 2009;123(2):729-738. https://doi.org/10.1097/PRS.0b013e3181958ec4

6. Akaike M, Fukotomi M, Nagamune M, et al. Simulation-based medical education in a clinical skills laboratory. J Med Invest 2012;59(1-2):28-35. https://doi.org/10.2152/jmi.59.28

Banks J, Carson JS, Nelson BL, Nicol DM. Introduction to simulation. In: Banks J, Carson JS, Nelson BL, Nicol DM. Discrete Event Simulation. Upper Saddle River: Pearson Education Inc., 2009:21.

8. Issenberg $\mathrm{SB}, \mathrm{McGaghie} W \mathrm{~W}$, Petrusa ER, et al. Features and Uses of High-fidelity Medical Simulations that Lead to Effective Learning: A BEME Systematic Review. BEME Guide No. 4. Best Evidence Medical Education. Dundee, Scotland: Association for Medical Education Europe (AMEE), 2004

9. Deziel DJ, Millikan KW, Economo SG, Doolas A, Ko ST, Airan MC. Complications of laparoscopic cholecystectomy: A national survey of 4292 hospitals and an analysis of 77604 cases. Am J Surg 1993;165(1):9-14 https://doi.org/10.1016/s0002-9610(05)80397-6

10. Haluck RS, Marshall RL, Krummel TM, Melkonian MG. Are surgery training programs ready for virtual reality? A survey of program directors in general surgery. J Am Coll Surg 2001;193(6):660-665. https://doi. org/10.1016/s1072-7515(01)01066-3

11. Accreditation Council for Graduate Medical Education (ACGME). Outcomes project. http:/www.acgme.org (accessed 26 June 2016).

12. Miller GE. The assessment of clinical skills/competence/performance. Acad Med 1990;65(9 Suppl):S63-S67. https://doi.org/10.1097/00001888-199009000-00045

13. Issenberg SB, McGaghie WC, Gordon DL, et al. Effectiveness of a cardiology review course for internal medicine residents using simulation technology and deliberate practice. Teach Learn Med 2002;14(4):223-228 https://doi.org/10.1207/S15328015TLM1404_4

14. Nel CPG, van Zyl GJ, Labuschagne MJ. Enhancement of plastic surgery training by including simulation in education and training. Afr J Health Professions Educ 2020;12(2):68-73. https://doi.org/10.7196/AJHPE.2020. v12i2.1182

15. Fouché CB. Research strategies. In: De Vos AS, Strydom H, Fouché CB, Delport CSL, eds. Research at Grass Roots: For the Social Sciences and Human Service Professions. 2nd ed. Pretoria: Van Schaik, 2002:270-277.

16. Greeff M. Information collection: Interviewing. In: De Vos AS, ed. Research at Grassroots. 2nd ed. Pretoria: Van Schaik, 2002:291-320.

17. Byrne M. Grounded theory as a qualitative research methodology. AORN J 2001;73(6):1155-1156. https://doi org/10.1016/S0001-2092(06)61841-3

18. Labuschagne MJ, Nel MM, Nel PPC, van Zyl GJ. Recommendations for the establishment of a clinical simulation unit to train South African medical students. Afr J Health Professions Educ 2014;6(2):2-6. https:// doi.org/10.7196/AJHPE.345

19. Nel CPG. Simulation in postgraduate plastic surgery education and training. PhD thesis. Bloemfontein: University of the Free State, 2019:42-43.

20. Ericsson KA. Deliberate practice and the acquisition and maintenance of expert performance in medicine and related domains. Acad Med 2004;79(10 Suppl.):S70-S81. https://doi.org/10.1097/00001888-200410001-00022 21. Satava RM. Emerging trends that herald the future of surgical simulation. Surg Clin North Am 2010;90(3):623-633 https://doi.org/10.1016/j.suc.2010.02.002

22. Nel CPG, Labuschagne MJ, van Zyl GJ. Simulation in plastic surgery: A research agenda to improve teaching learning and clinical expertise/professional competence. Ireland International Conference on Education, Dublin, Ireland, 25 - 28 April 2016. http://terezaventura.net/IICE-2016\%20Proceedings.pdf (accessed 4 November 2020).

23. Nel CPG, Labuschagne MJ, van Zyl GJ. Why is research needed on simulation to enhance plastic surgery education and training? Int J Cross Discpl Subject Educ 2018;1(1):3301-3308

Accepted 16 January 2020 